\title{
Fluid-Model-Based Car Routing for Modern Ridesharing Systems
}

\author{
Anton Braverman \\ Cornell University \\ ab2329@cornell.edu \\ Xin Liu \\ Arizona State University \\ xliu272@asu.edu
}

\author{
J.G. Dai \\ Cornell University \\ jim.dai@cornell.edu \\ Lei Ying \\ Arizona State University \\ lei.ying.2@asu.edu
}

\begin{abstract}
This paper considers a closed queueing network model of ridesharing systems such as Didi Chuxing, Lyft, and Uber. We focus on empty-car routing, a mechanism by which we control car flow in the network to optimize system-wide utility functions, e.g. the availability of empty cars when a passenger arrives. We establish both process-level and steady-state convergence of the queueing network to a fluid limit in a large market regime where demand for rides and supply of cars tend to infinity, and use this limit to study a fluid-based optimization problem. We prove that the optimal network utility obtained from the fluid-based optimization is an upper bound on the utility in the finite car system for any routing policy, both static and dynamic, under which the closed queueing network has a stationary distribution. This upper bound is achieved asymptotically under the fluid-based optimal routing policy. Simulation results with real-word data released by Didi Chuxing demonstrate that the utility under the fluid-based optimal routing policy converges to the upper bound with a rate of $1 / \sqrt{N}$.
\end{abstract}

\section{INTRODUCTION}

Because of the proliferation of ridesharing services, modeling and control of these systems have become important research topics over the last few years. This work focuses on empty-car routing mechanisms for controlling car flows in the network to optimize system-wide utility functions [2].

We consider a system with $r>0$ regions and $N>0$ cars. The regions can be interpreted as geographic regions in a city and cars drive around between regions transporting passengers. At time $t=0$, all cars start off idling empty in some region, waiting for a passenger. Passengers arrive to region $i$ according to a Poisson process with rate $N \lambda_{i}>0$, and arrivals to different regions are independent. When a passenger arrives to region $i$, if there is an empty car available there, then the passenger occupies that car and travels to region $j$ with probability $P_{i j}$. We allow $P_{i i}>0$ to represent trips within a region. Travel times from region $i$ to $j$ have mean $1 / \mu_{i j}$ and are assumed to be i.i.d. exponential random

Permission to make digital or hard copies of part or all of this work for personal or classroom use is granted without fee provided that copies are not made or distributed for profit or commercial advantage and that copies bear this notice and the full citation on the first page. Copyrights for third-party components of this work must be honored. For all other uses, contact the owner/author(s).

SIGMETRICS '17, fune 5-9, 2017, Urbana-Champaign, IL, USA

(c) 2017 Copyright held by the owner/author(s). ACM ISBN 978-1-4503-5032-7/17/06 DOI: http://dx.doi.org/10.1145/3078505.3078595 variables, although our main results hold without this assumption. If no empty car is available, the passenger abandons the system and finds an alternative form of transportation to the destination. Once the passenger arrives at region $j$, the car becomes empty. The empty car can either decide to stay in region $j$ (and wait for a new passenger) with probability $Q_{j j}$, or drive empty to a different region $k$ and wait for a passenger there with probability $Q_{j k}$. In general, the routing matrix (also called the routing policy) $Q=\left(Q_{i j}\right)$ is allowed to be state-dependent, i.e. $Q$ may depend on the current distribution of cars in the system. We assume $N, \lambda, \mu$, and $P=\left(P_{i j}\right)$ are given, and seek to choose $Q$ to optimize a system-wide utility function, e.g., to maximize system-wide availability of empty cars. To model these dynamics, we use a closed queueing network that belongs to the class of BCMP networks [1].

\section{THE RIDESHARING OPTIMIZATION PROBLEM}

In this section we introduce the fluid-based optimization and state our main results, Theorems 2.1 and 2.2. Let $E_{i j}^{(N)}(t)$ and $F_{i j}^{(N)}(t)$ be the number of empty and full cars en route from region $i$ to region, respectively, where $E_{i i}^{(N)}(t)$ is interpreted the number of empty cars waiting in region $i$ for a new passenger. Furthermore, define $\tilde{E}_{i j}^{(N)}(t)=\frac{1}{N} E_{i j}^{(N)}(t), \tilde{F}_{i j}^{(N)}(t)=\frac{1}{N} F_{i j}^{(N)}(t)$ and $A_{i}^{(N)}=$ $\mathbb{P}\left(\tilde{E}_{i i}^{(N)}(\infty)>0\right)$ to be the availability at region $i$. Let $\tilde{E}^{(N)}(t)$, $\tilde{F}^{(N)}(t)$ and $A^{(N)}$ be their corresponding matrix and vector versions.

We now consider the fluid-based optimization problem to be fully specified from (1) to (8) below. In the optimization problem, $c_{i}>0$ are rewards for picking up a passenger at region $i$, and $\tilde{c}_{i j}>0$ are costs of sending empty cars from $i$ to $j$. The variables in the optimization problem are $q, \bar{e}, \bar{f}, \bar{a}$. Let $q=\left(q_{i j}\right)$ be an $r \times r$ matrix representing a static empty-car routing policy $Q$. Let $\bar{e}, \bar{f}$, and $\bar{a}$ represent $\mathbb{E}\left[\tilde{E}^{(N)}(\infty)\right], \mathbb{E}\left[\tilde{F}^{(N)}(\infty)\right]$ and $A^{(N)}$ in the ridesharing network, respectively.

$$
\begin{array}{ll} 
& \max _{q, \bar{e}, \bar{f}, \bar{a}} \sum_{i=1}^{r} c_{i} \lambda_{i} \bar{a}_{i}-\sum_{i=1}^{r} \sum_{j=1, j \neq i}^{r} \tilde{c}_{i j} \bar{e}_{i j} \\
\text { subject to } \quad & \lambda_{i} P_{i j} \bar{a}_{i}=\mu_{i j} \bar{f}_{i j}, \\
& \mu_{i j} \bar{e}_{i j}=q_{i j} \sum_{k=1}^{r} \mu_{k i} \bar{f}_{k i},
\end{array}
$$




$$
\begin{aligned}
& \lambda_{i} \bar{a}_{i}=\sum_{k=1, k \neq i}^{r} \mu_{k i} \bar{e}_{k i}+q_{i i} \sum_{k=1}^{r} \mu_{k i} \bar{f}_{k i}, \\
& \left(1-\bar{a}_{i}\right) \bar{e}_{i i}=0, \\
& \sum_{i=1}^{r} \sum_{j=1}^{r} \bar{f}_{i j}+\sum_{i=1}^{r} \sum_{j=1}^{r} \bar{e}_{i j}=1, \\
& 0 \leq \bar{e}_{i j} \leq 1,0 \leq \bar{f}_{i j} \leq 1,0 \leq \bar{a}_{i} \leq 1, \\
& \sum_{j=1}^{r} q_{i j}=1, q_{i j} \geq 0 .
\end{aligned}
$$

The interpretation of (1)-(8) is intuitive. Objective (1) captures the tradeoff between revenue generation, and the cost of empty-car routing. Constraints (2)-(4) are flow-balance constraints. Constraint (5) states that for region $i$, either $\bar{a}_{i}=1$ or $\bar{e}_{i i}=0$, i.e. either availability is at $100 \%$ or the long-run fraction of empty cars at the station is zero.

The following are our main results. Theorems 2.1 establishes the connection between the fluid-based optimization problem and $\left(\tilde{E}^{(N)}, \tilde{F}^{(N)}\right)$. Theorems 2.2 shows that asymptotically, the optimal static policy from the fluid-based optimization outperforms all state dependent policies.

Theorem 2.1. Let $q, \hat{e}, \hat{f}, \hat{a}$ be a feasible solution to the optimization problem in (1)-(8). Set $Q=q$. Assume $P_{i j}>0$ for all $1 \leq i, j \leq r$ and $q_{i i}>0$ for all $1 \leq i \leq r$. Then

$$
\begin{aligned}
& \tilde{F}^{(N)}(\infty) \Rightarrow \hat{f}, \\
& \tilde{E}_{i j}^{(N)}(\infty) \Rightarrow \hat{e}_{i j}, 0 \leq i \neq j \leq 1, \\
& \tilde{E}_{i i}^{(N)}(\infty) \Rightarrow 0, \text { for } i \text { such that } \hat{a}_{i}<1, \\
& \sum_{i: \bar{a}_{i}=1} \tilde{E}_{i i}^{(N)}(\infty) \Rightarrow \sum_{i: \hat{a}_{i}=1} \hat{e}_{i i},
\end{aligned}
$$

and

$$
\mathbb{P}\left(E_{i i}^{(N)}(\infty)>0\right) \rightarrow \hat{a}_{i}, 0 \leq i \leq 1,
$$

as $N \rightarrow \infty$.

Theorem 2.2. (a) Suppose $\left(\tilde{E}^{(N)}, \tilde{F}^{(N)}\right)$ is irreducible under $P$ and $Q$, where $Q$ is a state-dependent empty-car routing policy. Let $\left(q^{*}, \bar{e}^{*}, \bar{f}^{*}, \bar{a}^{*}\right)$ be an optimal solution of the optimization problem in (1)-(8). Then

$$
\begin{aligned}
& \sum_{i=1}^{r} c_{i} \lambda_{i} A_{i}^{(N)}-\sum_{i=1}^{r} \sum_{j=1, j \neq i}^{r} \tilde{c}_{i j} \mathbb{E}\left[\tilde{E}_{i j}^{(N)}(\infty)\right] \\
& \leq \sum_{i=1}^{r} c_{i} \lambda_{i} \bar{a}_{i}^{*}-\sum_{i=1}^{r} \sum_{j=1, j \neq i}^{r} \tilde{c}_{i j} \bar{e}_{i j}^{*}, N>0 .
\end{aligned}
$$

(b) Let $\left(\tilde{E}^{(N) *}, \tilde{F}^{(N) *}\right)$ denote the CTMC under the static routing policy $q^{*}$. If $P_{i j}>0$ for all $1 \leq i, j \leq r$ and $q_{i i}^{*}>0$ for all $1 \leq i \leq r$, then

$$
\begin{aligned}
& \lim _{N \rightarrow \infty} \sum_{i=1}^{r} c_{i} \lambda_{i} A_{i}^{(N) *}-\sum_{i=1}^{r} \sum_{j=1, j \neq i}^{r} \tilde{c}_{i j} \mathbb{E}\left[\tilde{E}_{i j}^{(N) *(\infty)}\right] \\
& =\sum_{i=1}^{r} c_{i} \lambda_{i} \bar{a}_{i}^{*}-\sum_{i=1}^{r} \sum_{j=1, j \neq i}^{r} \tilde{c}_{i j} \bar{e}_{i j}^{*} .
\end{aligned}
$$

\section{EVALUATION}

In this section, we evaluate the convergence of the utility under the fluid-based policy to the fluid-based optimal solution, as $N$ increases, on a nine-region network as shown in Figure 1, which was extracted from a data set released in the Di-Tech Challenge by the Didi Research Institute http://research.xiaojukeji.com/index_ en.html. The nodes represent the regions, and the edge-weights are the estimated average travel times.

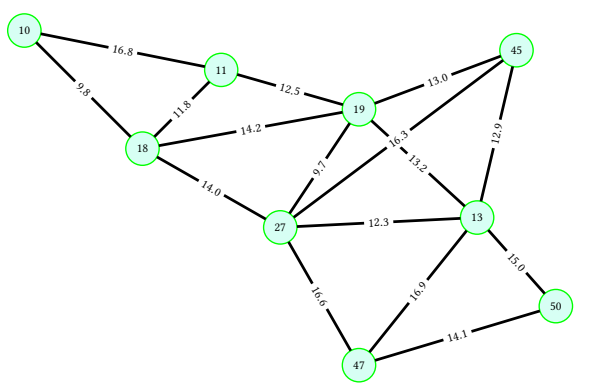

Figure 1: The nine-region network extracted from the DiDi dataset

We chose the utility function $U(\bar{a})=\frac{\sum_{i=1}^{r} \lambda_{i} \bar{a}_{i}}{\sum_{i=1}^{r} \lambda_{i}}$, which is the probability that a passenger requesting a ride at any region is fulfilled. The utility function $U(\bar{a})$ can be retrieved by setting $c_{i}=$ $\frac{1}{\sum_{i=1}^{r} \lambda_{i}}$ and $\tilde{c}_{i j}=0$ in (1). Let $Q^{*}$ be the optimal routing matrix from (2)-(8) with $U(\bar{a})$ and $a^{*}=U\left(\bar{a}^{*}\right)$ be the associated optimal system-wide availability. Let $a^{(N)}=U\left(A^{(N)}\right)$ be the system-wide availability for the finite sized system with $N$ cars under the optimal routing policy $Q^{*}$. Figure 2 shows that the convergence appears to be happening at a rate of $1 / \sqrt{N}$.

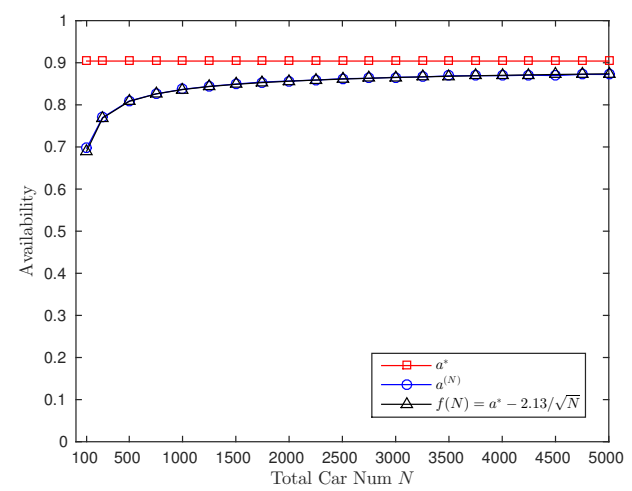

Figure 2: Convergence of the system-wide availability to its fluid-based optimal availability in the nine-region network.

\section{REFERENCES}

[1] F. Baskett, K. M. Chandy, R. R. Muntz, and F. G. Palacios. 1975. Open, closed and mixed networks of queues with different classes of customers. Fournal of the Association for Computing Machinery 22 (1975), 248-260.

[2] A. Braverman, J. G. Dai, X. Liu, and L. Ying. 2016. Empty-car routing in ridesharing systems. ArXiv e-prints (Sept. 2016). arXiv:math.PR/1609.07219 